Technological University Dublin ARROW@TU Dublin

\title{
Study of an Acrylamide-based Photopolymer for use as a Holographic Data Storage Medium
}

\author{
Hosam Sherif \\ Technological University Dublin \\ Izabela Naydenova \\ Technological University Dublin, izabela.naydenova@tudublin.ie \\ Suzanne Martin \\ Technological University Dublin, suzanne.martin@tudublin.ie
}

See next page for additional authors

Follow this and additional works at: https://arrow.tudublin.ie/cieocon2

Part of the Optics Commons

\section{Recommended Citation}

Sherif, H. et al. (2005) Study of an acrylamide-based photopolymer for use as a holographic data storage medium. SPIE proceedings of OPTO-IRELAND, V.5827, 305-313, doi:10.1117/12.604562 doi.org/

10.21427/r3tg-mc51

This Conference Paper is brought to you for free and open access by the Centre for Industrial and Engineering Optics at ARROW@TU Dublin. It has been accepted for inclusion in Conference Papers by an authorized administrator of ARROW@TU Dublin. For more information, please contact arrow.admin@tudublin.ie, aisling.coyne@tudublin.ie,gerard.connolly@tudublin.ie.

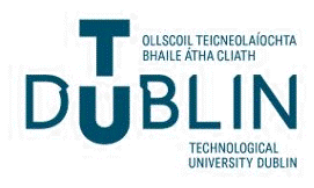




\section{Authors}

Hosam Sherif, Izabela Naydenova, Suzanne Martin, Colm McGinn, G Berger, C Denz, and Vincent Toal 
2005-01-01

\section{Study of an acrylamide-based photopolymer for use as a holographic data storage medium}

Hosam Sherif

Dublin Institute of Technology

Izabela Naydenova

Dublin Institute of Technology, izabela.naydenova@dit.ie

Suzanne Martin

Dublin Institute of Technology, suzanne.martin@dit.ie

C. McGinn

Dublin Institute of Technology

G. Berger

Wilhelms-Universitaet Muenster

See next page for additional authors

\section{Recommended Citation}

H. Sherif, I. Naydenova, S. Martin, C. McGinn V. Toal, G. Berger, C. Denz "Study of an acrylamide-based photopolymer for use as a holographic data storage medium" SPIE proceedings of OPTO-IRELAND, V.5827, 305-313, 2005. doi:10.1117/12.604562

This Conference Paper is brought to you for free and open access by the Centre for Industrial and Engineering Optics at ARROW@DIT. It has been accepted for inclusion in Articles by an authorized administrator of ARROW@DIT. For more information, please contact

yvonne.desmond@dit.ie, arrow.admin@dit.ie. 
Authors

Hosam Sherif, Izabela Naydenova, Suzanne Martin, C. McGinn, G. Berger, C. Denz, and Vincent Toal 


\title{
Study of an acrylamide-based photopolymer for use as a holographic data storage medium
}

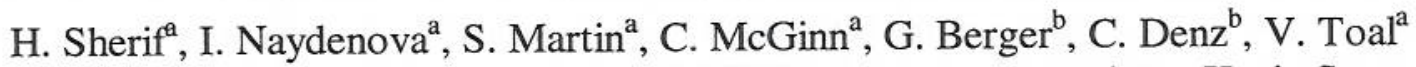 \\ ${ }^{a}$ Centre for Industrial and Engineering Optics, Dublin Institute of Technology, Kevin Street, Dublin \\ 8, Ireland. \\ 'Institute for Applied Physics, Westfaelische, Wilhelms-Universitaet Muenster, Corrensstr. 2/4, \\ 48149 Muenster, Germany.
}

Tel: 00353-1- 4027927 Fax: 00353-1- 4027901

Email: hosam.sherif@student.dit.ie

\begin{abstract}
An acrylamide-based photopolymer formulated in the Centre for Industrial and Engineering Optics has been investigated with a view to further optimisation for holographic optical storage. Series of 15 to 30 gratings were angularly multiplexed in a volume of the photopolymer layers with different thickness at a spatial frequency of $1500 \mathrm{lines} / \mathrm{mm}$. Since the photopolymer is a saturable material, an exposure scheduling method was used to exploit the entire dynamic range of the material and allow equal strength gratings to be recorded. From this investigation the photopolymer layer's $\mathrm{M} / \#$ was determined with regard to the recording geometry used. The temporal stability of photopolymer layers was studied in terms of diffraction efficiency and change of the reconstructed angle due to material shrinkage. In addition, the potential of the photopolymer as a holographic data storage medium was demonstrated by recording bit data-pages.
\end{abstract}

Keywords: holographic material, photopolymer dynamic range, holographic characteristics, shrinkage

\section{INTRODUCTION}

Holographic data storage gives rise to potential capacities of $1 \mathrm{~TB} / \mathrm{cm}^{3}$ with transfer rates of $10 \mathrm{~GB} / \mathrm{s}^{1-3}$. This is primarily due to the information being stored in the volume of the recording material as a series of data pages. The pages are multiplexed (superimposed) into the volume and allow parallel recording and readout of data, thus achieving high data transfer rates with short access times. In recent times holographic data storage HDS systems and storage media have progressed from demonstration set ups to being available commercially, with companies such as InPhase Technologies, Optware, and Aprilis all making this transistion ${ }^{4-5}$. There is still need for further improvement, with emphasis being shifted to investigating different materials to find the optimum medium for this new technology.

This investigation is focused on the characterisation of a photopolymer system to determine its suitability for use as a HDS media. The material's dynamic range was studied, this being the total response of the material and capability of storing multiple gratings in the same volume. A study of the material's temporal stability was also carried out, this included monitoring the material shrinkage, changes in the Bragg selectivity curve and diffraction efficiency.

Finally, bit data pages were recorded to ascertain photopolymer characteristics such as the recording exposure energies required to store data pages with low bit error rate (BER), longevity of unfixed data pages i.e. available recording period. A qualitative assessment of these parameters is presented in this report. The characterisation was conducted in the Institute for Applied Physics, Westfaelische, Wilhelms-Universitaet, Muenster.

The material chemistry and layer preparation have thus far been optimised for applications other than HDS, these include real time holographic interferometry ${ }^{6}$, electronic speckle pattern interferometry ${ }^{7}$, and holographic optical elements ${ }^{8}$. The purpose of this work was assessment of the material's performance in its current formulation for moderately thick layers of 100-160 $\mu \mathrm{m}$ thickness. 


\section{PHOTOPOLYMER CHEMISTRY}

The acrylamide-based photopolymer is a self-developing dry layer. It consists of a polyvinylalcohol binder in which a monomer, an electron donor, and dye sensitizer (erythrosine B) are dissolved. When a dye molecule absorbs a photon in the presence of an electron donor, free radicals are produced that cause local polymerisation of the acrylamide. A corresponding variation occurs in the local refractive index of the material ${ }^{9}$. This variation is not only attributed to bond conversion in the polymerisation process, but also to the secondary effect, whereby the local density is modulated as mass transport occurs during the recording. The photopolymerisation process starts when an erythrosine B molecule (XD) absorbs a photon of light and is promoted to an excited singlet state.

$$
\mathrm{XD}+h v={ }^{1} \mathrm{XD} *(\text { absorption of light) }
$$

It may then transfer to an excited triplet state through intersystem crossing.

$$
{ }^{1} \mathrm{XD} *={ }^{3} \mathrm{XD} * \text { (intersystem crossing) }
$$

Then it may react with the electron donor (ED), triethanolamine, to produce a dye radical and a triethanolamine free radical.

$$
{ }^{3} \mathrm{XD} *+\mathrm{ED}=\mathrm{XD} \bullet+\mathrm{ED} \bullet(\text { dye reduction-electron transfer) }
$$

By itself, the dye radical is not normally reactive enough to initiate polymerisation. The polymerisation is usually due to the triethanolamine radical reacting with an acrylamide molecule (ACR):

$$
\begin{aligned}
& \mathrm{ED} \bullet+\mathrm{ACR}=\mathrm{ED}-\mathrm{ACR} \bullet(\text { initiation}) \\
& \mathrm{ED}-\mathrm{ACR} \bullet+\mathrm{ACR}=\mathrm{ED}-(\mathrm{ACR}) 2^{\bullet} \text { (propagation) }
\end{aligned}
$$

\section{EXPERIMENTAL}

A plane wave recording system utilizing angular multiplexing was constructed, Fig. 1, having a rotational stage whose axis is at the intersection of the object and reference beams, and in the plane of the photosensitive layer. An $\mathrm{Nd} \mathrm{YVO}_{4}$ (Verdi V-5) $532 \mathrm{~nm}$ laser beam was spatially filtered using a 10x objective lens and a $15 \mu \mathrm{m}$ pinhole. The filtered beam was then collimated before being split into two equally intense beams of $10 \mathrm{~mm}$ diameter. The angle between the beams was set to approximately $47^{\circ}$. This produced holographic gratings with a spatial frequency of $1500 \mathrm{lines} / \mathrm{mm}$. Storing multiple gratings in the same volume entailed rotating the photopolymer layer by an angle of $1.5^{\circ}$ between recordings. This angle was chosen so that the first order diffracted peak of each grating would not contribute to or detract from the diffraction efficiency of neighbouring gratings. A combined exposure intensity of $3.2 \mathrm{~mW} / \mathrm{cm}^{2}$ was used to record the individual gratings, the exposure energy varied for each grating due to the different scheduling times being used.

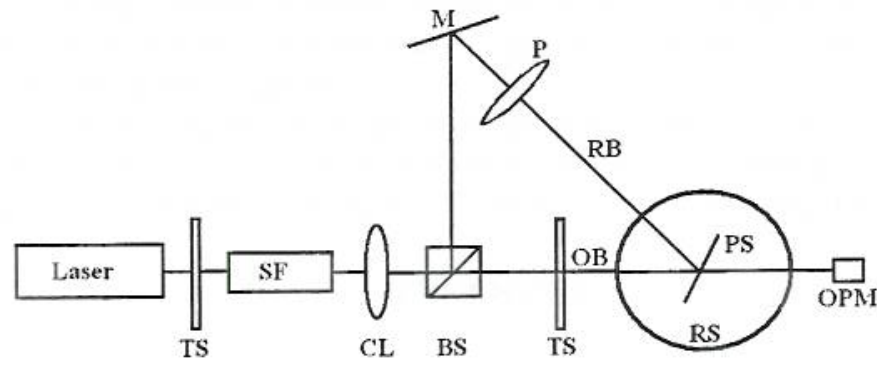

Fig. 1 Experimental set-up for characterisation of acrylamide based photopolymer, TS: shutter, SF: spatial filter, CL: collimating lens, BS: beam splitter, P: polarizer, RS: rotational stage, PS: photopolymer holder OPM: optical power meter, M: mirror, RB: reading beam, OB: object beam. 
The stored holographic gratings were readout with the same wavelength laser as used to record them. The reference beam power was reduced to $\approx 128 \mu \mathrm{W} / \mathrm{cm}^{2}$ so that additional photopolymerisation of the material would be negligible at readout.

\section{DETERMINATION OF M/\#}

The photopolymer's dynamic range is not shared equally among the multiplexed gratings when they are recorded with equal exposure energy; this is due to the photopolymer being a saturable material. This means that as additional gratings are multiplexed in the volume they have less potential for modulating the materials refractive index. The grating diffraction peaks are expected to scale ${ }^{10}$ as $1 / \mathrm{N}^{2}$. Consequently, as the number of gratings multiplexed increases, it becomes desirable to equalise the diffraction peaks so that they all have the same signal-to-noise ratio. To do this an iterative exposure scheduling method ${ }^{11}$ is used to equally share the dynamic range between all of the multiplexed gratings.

The material's M/\# was determined for layers $\approx 160 \mu \mathrm{m}$ thick by first multiplexing series of 18 to 30 holographic gratings in the photopolymer layer. With each additional grating angularly multiplexed into the volume the angle between the photopolymer layer and the bisector of the recording beams becomes smaller. It is for this reason that only 30 gratings were recorded as more than this would result in high Fresnel reflection losses. The recording system is being modified so that it will utilize peristrophic as well as angular multiplexing; in future, this should increase the number of gratings that can be recorded. To reduce the inhibition period, i.e. the time it takes for the concentration of an inhibitor molecule, oxygen in this case, to fall below a critical value whereby the polymerization process can begin ${ }^{12}$, a preexposure of $480 \mu \mathrm{J} / \mathrm{cm}^{2}$ was used before the multiplexed gratings were recorded. This energy was delivered to the material through one beam only. A delay of 100 seconds was then used to allow for all the preexposure processes within the material to come to a halt.

\section{RESULTS AND DISCUSSION}

Recording gratings with a constant two second exposure produced holographic gratings of highly differing strengths. The majority of the diffracted energy was consumed in the first five gratings leaving little dynamic range for subsequent gratings. When considering HDS it is advantageous to multiplex as many holograms as possible, each with the same diffraction efficiency as its neighbour and having a significantly large signal-to-noise ratio so as to be easily read back out of the storage medium. With this in mind, it becomes clear that a large series of weaker but equalised holograms is more desirable than recording a small number of unequalised holograms.

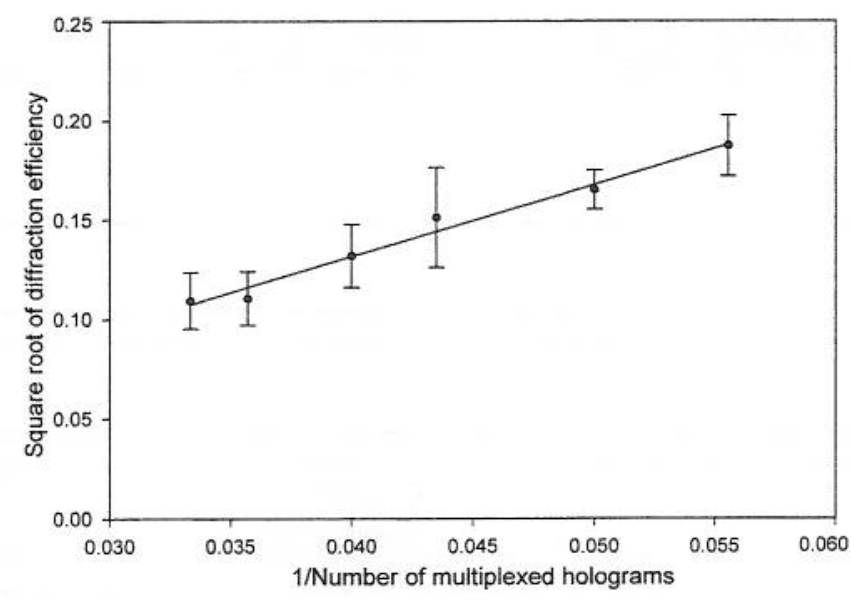

Fig. 2 The square root of diffraction efficiency as a function of the reciprocal of number of gratings multiplexed yields an $\mathrm{M} / \#=3.6$ for layers $\approx 160 \mu \mathrm{m}$ thick. 
New gratings are multiplexed in the photopolymer this time with the new exposure times that have been determined after applying the proposed algorithm ${ }^{11}$. With each exposure scheduling iteration, the reconstructed gratings exhibit an increase in the uniformity of the diffraction efficiencies.

A plot of the square root of the mean diffraction efficiency as a function of the reciprocal of the number of gratings can be seen in Fig. 2. The slope of this graph produces an $\mathrm{M} / \#$ of 3.6 for layers $\approx 160 \mu \mathrm{m}$ thick. This compares favorably to the two-chemistry system (formulations 523530 and 323530) developed by InPhase Technology ${ }^{4}$. The quoted M/\# values are taken based on $200 \mu \mathrm{m}$ samples. In the case of 523530 the $\mathrm{M} / \#$ is 6.7 and the shrinkage is $0.1 \%$ whereas 323530 has a higher $\mathrm{M} / \#$ of 8.7 but also a higher shrinkage of $0.5 \%$. Thicker samples of $1.5 \mathrm{~mm}$ produce $\mathrm{M} / \#$ of 50 and 45 respectively ${ }^{4}$. The $\mathrm{M} / \#$ for our material is still unknown at these higher layer thicknesses but It is reasonable to assume that it will be higher. Work is currently being carried out to produce thicker photopolymer samples, which have good optical quality.

\section{TEMPORAL STABILITY STUDY}

To determine whether the material undergoes any form of mechanical deformation such as shrinkage, a slanted grating was recorded in the photopolymer layer. Shrinkage in a slanted grating would result in a drop off in diffraction efficiency of the reconstructed grating or worse, no diffraction of the signal beam at the expected reconstruction angle. The reason for this is that the slant angle of the grating fringes changes, leading to a corresponding change in the fringe period. To reconstruct the grating with maximum diffraction efficiency it would then be required that either the wavelength or the reference beam angle be readjusted.

The stability of the photopolymer layer can also be affected by undesired diffusion and polymerisation of the acrylamide monomer after recording. This could also result in a reduction in the diffraction efficiency or have an adverse effect on the reconstructed Bragg selectivity curve. A HDS medium that experiences a high level of shrinkage after recording will exhibit a decrease in signal-to-noise ratio and consequently an increase in bit error rate of the recorded data.

\subsection{Shrinkage}

The photopolymer shrinkage was determined by recording a single slanted holographic grating in a layer approximately $120 \mu \mathrm{m}$ thick. A slant angle of $20^{\circ}$ was used to record this grating. This was taken to be the angle between the photopolymer layer normal and the bisector of the recording beams. A spatial frequency of $1500 \mathrm{lines} / \mathrm{mm}$ and exposure energy of $23 \mathrm{~mJ} / \mathrm{cm}^{2}$ was used to record the grating. An $\mathrm{Ar}^{+} 514 \mathrm{~nm}$ wavelength laser was used to deliver this exposure energy to the grating. Once the grating had been recorded, it was probed with a wavelength of $514 \mathrm{~nm}$ and an intensity of $59 \mu \mathrm{J} / \mathrm{cm}^{2}$. The layer itself was sealed between two glass plates before recording. The grating's diffracted light intensity was angularly scanned. A grating of low diffraction efficiency was recorded; this is typical of one of a set of multiplexed holograms.

The occurrence of shrinkage of the photopolymer layer results in a change in the fringe spatial period ${ }^{13}$. To achieve maximum diffraction efficiency it is necessary to adjust the readout beam angle. By knowing both the initial slant angle of the grating $\phi_{0}$ and the final slant angle $\phi_{1}$ the fractional change in material thinckness, $\Delta d$, can be obtained.

$$
\Delta d=d_{0}\left[\frac{\tan \phi_{1}}{\tan \phi_{0}}-1\right]
$$




\subsection{Bragg selectivity curve and diffraction efficiency}

Examining the grating over the same 37 day period showed that the replay angle of the slanted grating had shifted from the angle at which it had been recorded, Fig. 3. This shift in replay angle is ascribed to layer shrinkage and the corresponding decrease in slant angle of the grating. While the replay angle changes over the period of investigation, there is no significant change in the shape of the Bragg selectivity curve; however the diffraction efficiency varied by $11 \%$ between the initial and the final angular scan.

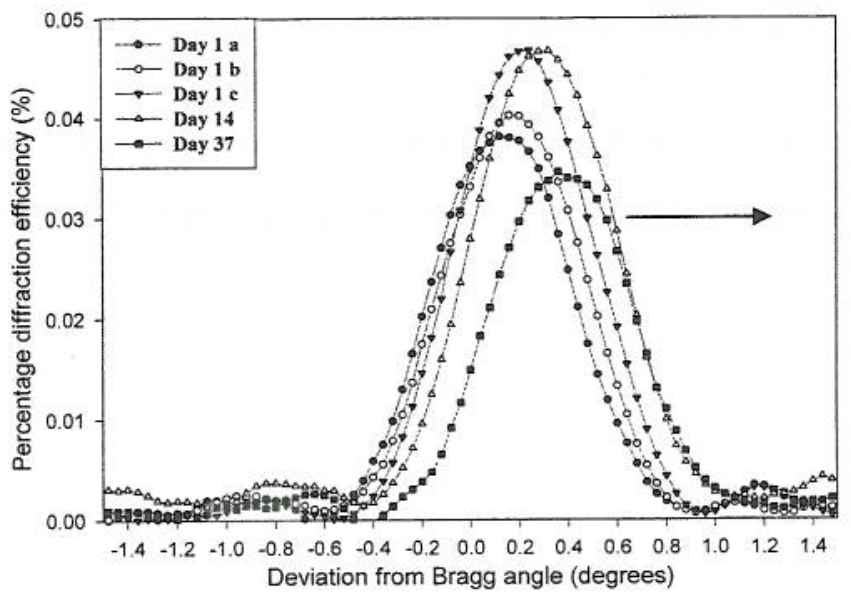

Fig. 3 Variation in the Bragg replay angle of the slanted grating during the period of investigation. The arrow indicates the direction of shrinkage.

\section{BIT DATA PAGE RECORDING}

\subsection{Experimental work}

A HDS system built at the Wilhelms-Universität Muenster, which works on the principal of phase-code multiplexing ${ }^{14}$, was used to characterise the acrylamide-based photopolymer in terms of its ability to store bit data pages, see Fig. 4. A $488 \mathrm{~nm}$ blue laser was used for recording and replaying the data pages. Positioned immediately after the laser were a $\lambda / 2$ plate and a polarising beam splitter, thus creating a signal and reference arm where the beam intensity ratio between both arms of the set-up could be adjusted. A beam expander and mirror configuration was used in each arm of the system to increase the size of the Gaussian profile of the laser beam. The central parts of the beams were then reflected by $90^{\circ}$ towards each other to interfere. With this arrangement, the homogeneity of the beams that eventually pass through the phase-code modulator and the SLM is increased significantly. This is vital for recording and playback of data pages with low BER. The expanded beam in the signal arm is collimated before entering the SLM. A polariser is placed between the collimating lens and the SLM to ensure the incident beam is vertically polarised. A second polariser is placed immediately after the SLM only allowing horizontally polarised light to pass through it. This is then rotated by $90^{\circ}$ to its initial vertical polarisation state by means of a $\lambda / 2$ plate. A converging lens is finally used to reduce the image from the SLM so that it passes through the Fourier plane before being imaged on to a CCD camera. A $4 \mathrm{~mm} \times 4 \mathrm{~mm}$ square iris diaphragm was placed in the beam path to allow only the central part of the Fourier image to be permitted to pass through to the photopolymer layer. 


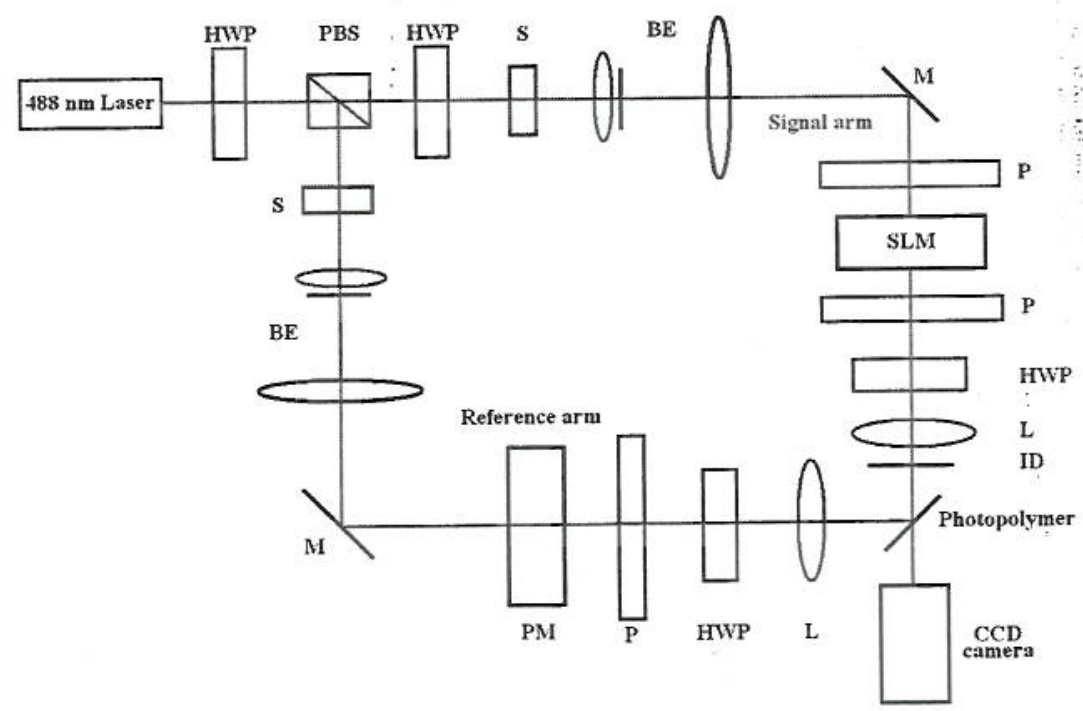

Fig. 4 Experimental set-up used for recording data pages $(H W P=\lambda / 2$ plate, $P B S=$ polarising beam splitter, $S=$ electronic shutters, $B E$ = beam expanders, $\mathrm{M}=$ mirrors, $\mathrm{SLM}=$ spatial light modulator, $\mathrm{L}=$ lens, $\mathrm{ID}=$ iris diaphragm,

$\mathrm{P}=$ polarisers, $\mathrm{PM}=$ phase modulator)

The photopolymer layer was placed perpendicular to the surface of the bench. It was adjusted so it made an angle of $35^{\circ}$ to the signal beam and $55^{\circ}$ to the reference arm. These angles were chosen as opposed to a $45^{\circ}$ angle between each beam, because interference fringes from the glass slide were being observed. As these were of comparable size to the imaged data page, it was necessary to remove them by using asymmetrical angles of incidence. The area of overlap on the photopolymer between the incident beams occurs in the Fresnel plane, approximately $2 \mathrm{~mm}$ in front of the Fourier plane of the signal beam. A converging lens was also used in the reference arm. A highly homogeneous region of the reference beam was overlapped with the signal beam, thus minimising the effect of an uneven beam profile. The signal and reference arm intensities were made equal by rotating the first half wave plate.

\subsection{Recording and playback of a data page}

Each data page was recorded in the photopolymer layer as a modulation of the refractive index of the material resulting from a complex interference pattern. The data pages themselves consisted of a random two-dimensional array of light and dark squares corresponding to the binary numbers 1 and 0 and having an ratio of 1000:1 between their respective intensities. The same data page was used for all experiments conducted thus maintaining consistency between experiments. It was digitally sent to the SLM as a standard $800 \times 600$ VGA image. The bits constituting the data page were oversampled so that every block of $10 \times 10$ pixels on the SLM corresponded to a single bit of data; therefore the final image consisted of 80 horizontal bits by 60 vertical bits. When imaged onto the photopolymer the page measured 1 $\mathrm{mm}$ by $0.75 \mathrm{~mm}$ with each bit measuring $12.5 \mu \mathrm{m}$ on its side. The duration of recording was determined by computer controlled shutters.

For the majority of experiments a combination of neutral density filters was used to attenuate the readout beam by $74 \%$ thus reducing the possibility of further polymerisation of the layer. When determining the recording time (see section 8.4 ) of the photopolymer only $0.6 \%$ of the reference beam intensity was used to playback the recorded holographic data page each time it was monitored. This was done to reduce the effect of post polymerisation of the recorded hologram over the course of the investigation.

\subsection{Optimisation of recorded data pages}

A series of data pages were recorded with exposure energies ranging from $4 \mathrm{~mJ} / \mathrm{cm}^{2}$ to $12 \mathrm{~mJ} / \mathrm{cm}^{2}$. For each exposure energy a number of different combinations of the exposure time and intensities were used to record the pages. Data pages of consistently high quality were observed when recorded with $2.8 \mathrm{~W} / \mathrm{cm}^{2}$ for 2.8 seconds. These pages were observed to have high white/black bit contrast with well-defined edges and good image resolution. It is assumed that the volume of 
photopolymer that stores the data could be reduced even further and still provide faithful reconstruction of the data. This can only be confirmed once a quantitative study of how the BER increases as data page size decreases is made.

Fig. 5(a) shows the data page imaged on to the SLM. The same page was also used for examining the recording period of the photopolymer. An analogue image, Fig. 5(b), was also imaged on the SLM and then holographically recorded using the optimal exposure time and intensity to test the materials response to an image containing a large amount of spatial frequencies. Figures 5(c) and 5(d) show the reconstructions of the recorded holograms.

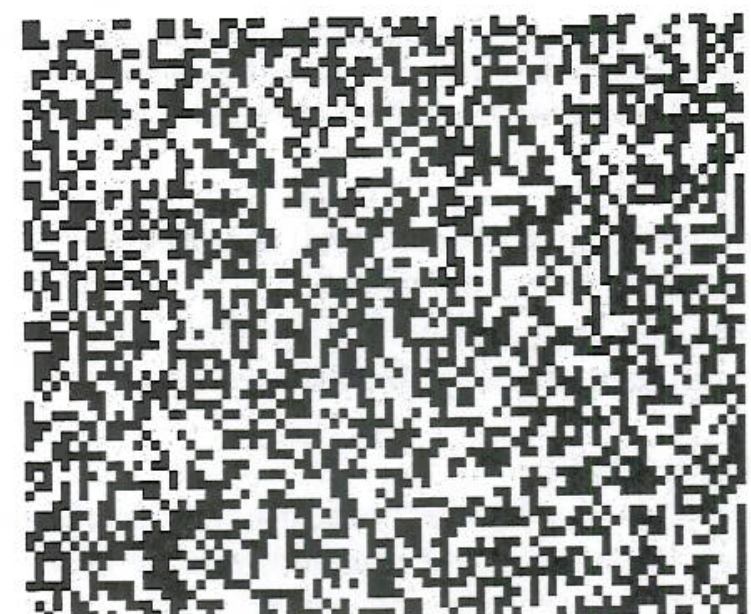

Figure 5(a)

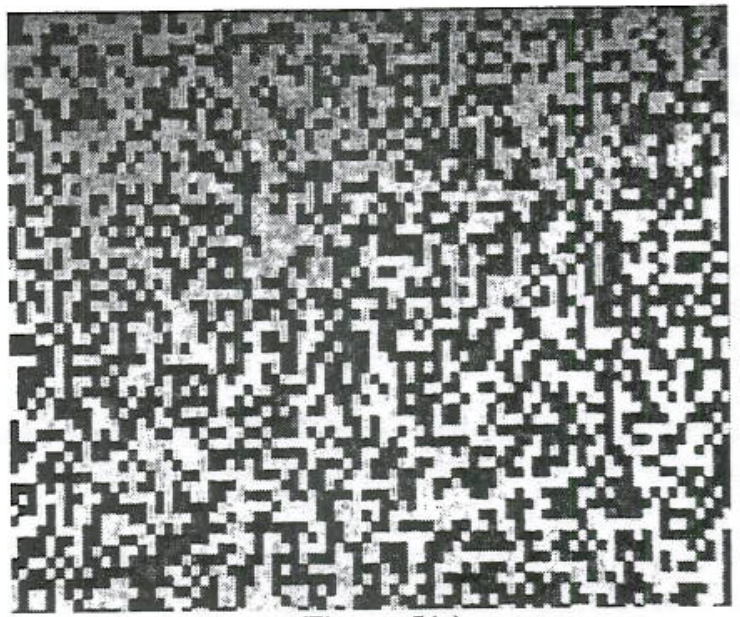

Figure 5(c)

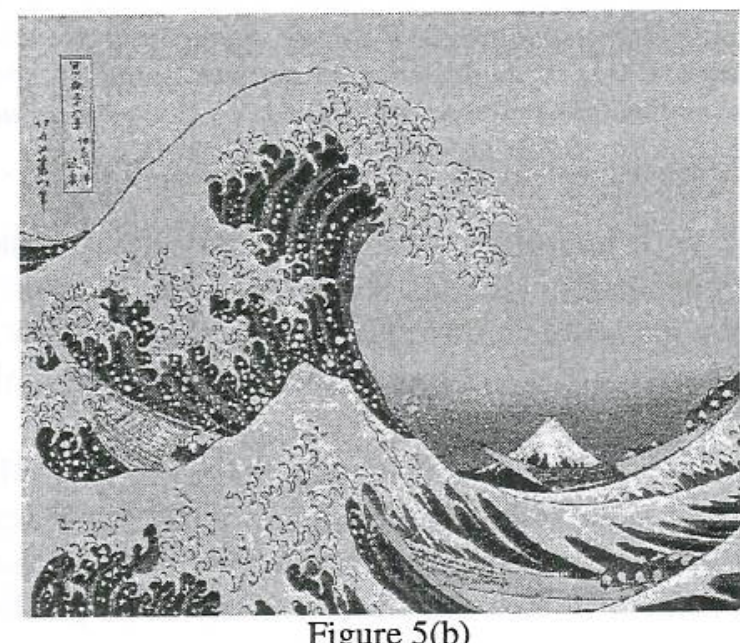

Figure 5(b)

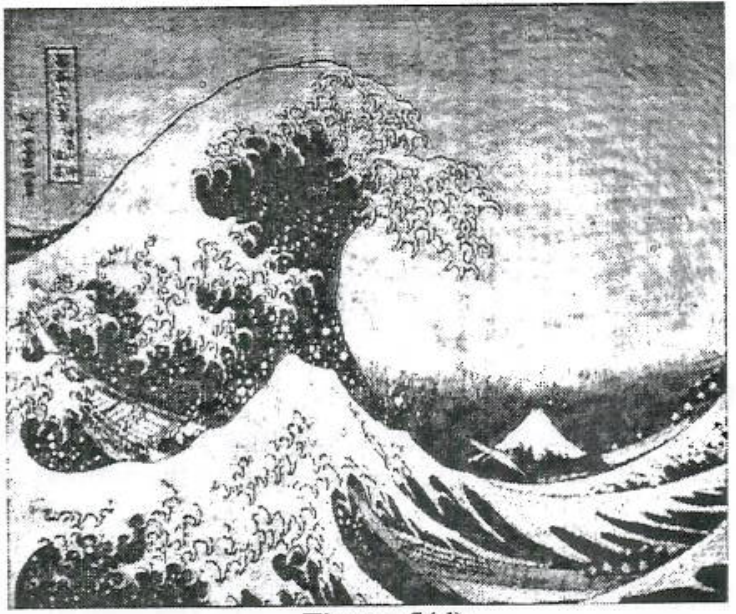

Figure 5(d)

Fig. 5 Image of data page and analogue image as they appeared on the SLM (a) \& (b). The reconstructed holographic images which were produced with a combined beam exposure energy of $8 \mathrm{~mJ} / \mathrm{cm}^{2}$ (c) \& (d).

\subsection{Determination of recording period}

With regard HDS, the period available to record data pages with low BER, after initial photopolymerisation, is an important material parameter. All holographically multiplexed data should be recorded during this period. The photopolymer can then be fixed to inhibit further photopolymerisation by consuming the entire erythrosine B dye molecule and so halting further creation of free radicals. 


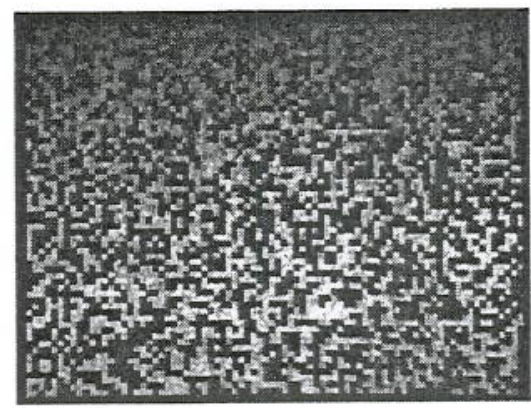

Figure 6(a) 20 seconds

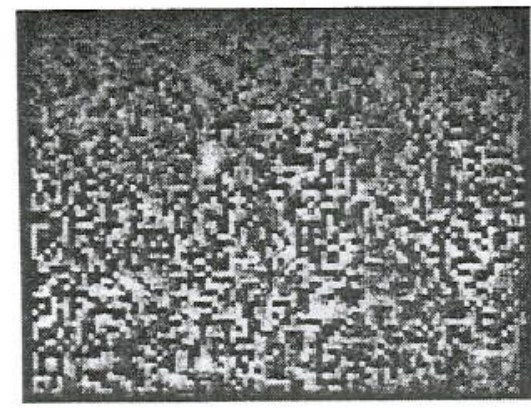

Figure 6(d) 1 hours

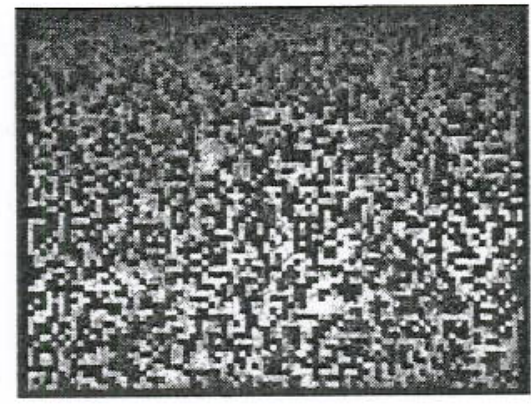

Figure 6(b) 10 minutes

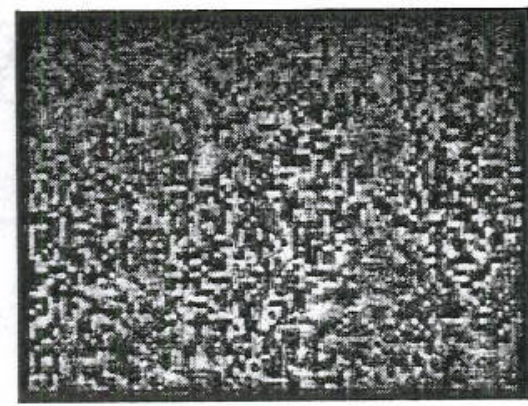

Figure 6(e) 4 hours

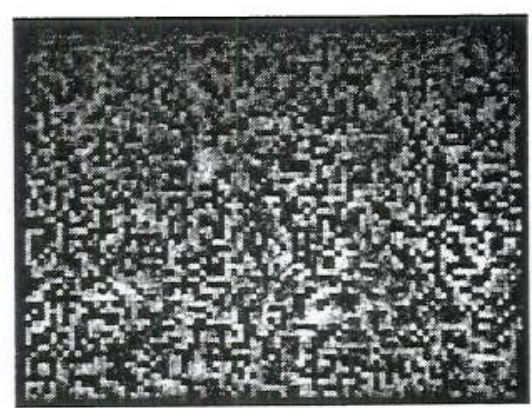

Figure 6(c) 30 minutes

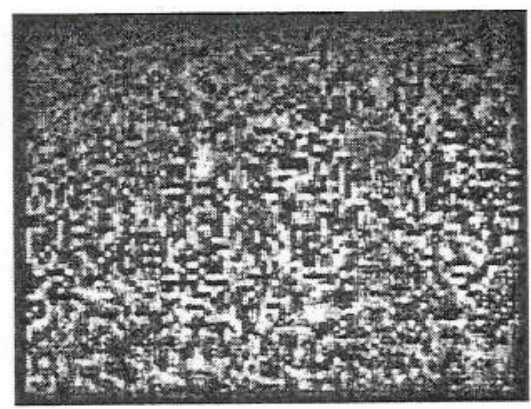

Figure 6(f) 8 hours

Fig. 6 Images (a) to (f) show a reconstructed holographic data page at different intervals over a period of 20 seconds to 8 hours after initial recording. A degradation of image quality is observed.

It is seen in Fig. 6 that after 1 hour it was observed that there was significant degradation of the data page in terms of a decrease in diffraction efficiency in certain regions of the image and smearing of the well-defined borders between individual bits.

The observed change in the recorded hologram is attributed to the diffusion of material between exposed and unexposed regions of the photopolymer. Prior to this investigation, it was observed that two different diffusion processes take place during and after holographic recording in the photopolymer system. The first process occurs when the monomer molecule diffuses from dark to bright fringe areas of the photopolymer, resulting in a positive contribution to the final refractive index modulation. The second process involves diffusion of terminated or unterminated short polymer chains from bright to dark fringe regions. This has a negative contribution to the final diffraction efficiency ${ }^{15}$.

\section{SUMMARY}

A novel acrylamide-based photopolymer system was characterised by means of a recording system that angularly multiplexed gratings into the same volume of the photopolymer. Sets of 18 to 30 multiplexed gratings were equalized and from the results obtained the material's M/\# for the particular geometry used was calculated to be 3.6 for a photopolymer layer $160 \mu \mathrm{m}$ thick.

The photopolymer's temporal stability was studied by recording a slanted grating and monitoring how its replay angle varied over a 37 day period. It was found that initially the material shrank by $1 \%$ after the first day; eventually reaching a value of $2 \%$ at day 37. Compared with the quoted values for InPhase Technology's photopolymers (523530 and 323530 , $0.1 \%$ and $0.5 \%$ respectively) the measured shrinkage is quite large. The diffraction efficiency of the same slanted grating varied by $11 \%$ of its original diffraction efficiency between the initial and the final angular scan made. It was noted that there was no significant change in the shape of the Bragg selectivity curve over period of investigation. 
Finally, the photopolymers ability to record and store bit wise data pages was evaluated. The optimal exposure intensity and exposure time needed to record a single holographic data page with good quality were determined to be $2.8 \mathrm{~mW} / \mathrm{cm}^{2}$ for 2.8 seconds. It was found that exposure energies ranging from $6-12 \mathrm{~mJ} / \mathrm{cm}^{2}$ can be used to record good quality data pages. An eight hour study was then carried out to determine the recording period of a single data page stored in the material prior to any fixing of the sample. The fidelity of the data pages was found to decrease over an eight hour interval. It was observed that good quality image was sustainable for up to 1 hour after initial recording had begun.

\section{ACKNOWLEDGMENTS}

The authors would like to acknowledge COST Action P8 "Materials and Systems for Optical Data Storage and Processing" for funding the short-term scientific mission of Hosam Sherif at the group of Prof. Dr. C. Denz, Institute for Applied Physics, Wilhelms-Universitaet Muenster.

"Focas Institute" is funded under the Programme for Research in Third Level Institutions (PRTLI).

\section{REFERENCES}

[1] P. Hariharan, "Optical Holography - Principles, Techniques, and Applications", 2nd ed. Cambridge University Press, Cambridge, UK, 1996.

[2] S. S. Orlov, W. Phillips, E. Bjornson, Y. Takashima, P. Sundaram, L. Hesselink, R. Okas, D. Kwan, R. Snyder, "High-transfer-rate high-capacity holographic disk data-storage system", App. Opt. 43(25), 4902-4914, Sept. 2004.

[3] G. W. Burr, J. Ashley, H. Coufal, R. K. Grygier, J. A. Hoffnagle, C. M. Jefferson, B. Marcus, "Modulation coding for pixel-matched holographic data storage", Opt. Lett. , 22(9), 639-641, May 1997.

[4] M. Schnoes, B. Ihas, A. Hil, L. Dhar, D. Michaels, S. Setthachayanon, G. Schomberger and W. L. Wilson "Holographic Data Storage Media for Practical Systems", www.inphase-technologies.com/technology/whitepapers

[5] R. T. Ingwall, D. A. Waldman, “CROP photopolymers for hologram recording”, SPIE, Holography, 11(2), 1-7, Dec. 2000.

[6] E. M. Mihaylova, V. Toal, S. R. Guntaka, S. Martin, "Detection of cracks and defects using electronic speckle pattern interferometry with a holographic optical element", Pub: Proc. SPIE Vol. 5226, 209-213, 12th International School on Quantum Electronics: Laser Physics and Applications; P. A. Atanasov, A. A. Serafetinides, I. N. Kolev; Eds: Nov. 2003.

[7] E. Mihaylova, I. Naydenova, H. Sherif, S. Martin, V. Toal, "Application of photopolymer holographic gratings in electronic speckle pattern shearing interferometry", Proc. of SPIE, 5249, Optical Systems Design and Engineering, 318326, L. M. Philip R., R. Wartmann, Editors, Saint-Etienne, Feb. 2003.

[8] S. R. Guntaka, V. Toal, S. Martin, "Holographically recorded diffraction optical elements for holographic and Electronic Speckle Pattern Interferometry”, App. Opt. 41(35), 7475-7479, Dec. 2002.

[9] S. Martin, P. Leclere, Y. Renotte, V. Toal, Y. Lion, "Characterisation of an acrylamide-based dry photopolymer holographic recording material”, Opt. Eng. 33 3942, Sept. 1994.

[10] G. W. Burr, D. Psaltis, "System metric for holographic memory systems", Opt. Letts. 21( 12), 896-898, June 1996.

[11] A. Pu, K. Curtis, D. Psaltis, "Exposure Schedule for multiplexing holograms in photopolymer films", Opt. Eng. 35

(10) 2824-2829, Oct. 1996.

[12] T. V Galstian, S. Harbour, A. V Galstyan, R. S Hakobyan, "Study of the inhibition period prior to the holographic grating formation in liquid crystal photopolymerizable materials", electronic-Liquid Crystal Communications, http://www.e-lc.org/docs/2004_05_05_11_13_17, May 2004.

[13] F.T. O'Neill, J.R. Lawrence, J.T. Sheridan, "Thickness variation of self-processing acrylamide-based photopolymer and reflection holography", Opt. Eng. 40(4) 533-53, April 2001.

[14] K. -O. Müller, C. Denz, T. Rauch, T. Heimann, T. Tschudi, "High capacity holographic data storage based on phase-coded multiplexing”, Optical memory and neural networks, 7(1) 1-10, 1998.

[15] I. Naydenova, R. Jallapuram, R. Howard, S. Martin, V. Toal, "Investigations of the diffusion processes in selfprocessing acrylamide-based photopolymer system" App. Opt. 43(14), 2900, 2004. 